\title{
Editorial
}

\section{Qualitative Theory of Differential, Difference, and Dynamic Equations}

\author{
Tongxing Li, ${ }^{1}$ Tuncay Candan, ${ }^{2}$ and Ethiraju Thandapani ${ }^{3}$ \\ ${ }^{1}$ Qingdao Technological University, Feixian, Shandong 273400, China \\ ${ }^{2}$ Department of Mathematics, Faculty of Arts and Sciences, Niğde University, 51200 Niğde, Turkey \\ ${ }^{3}$ Ramanujan Institute for Advanced Study in Mathematics, University of Madras, Chennai 600 005, India
}

Correspondence should be addressed to Tongxing Li; litongx2007@163.com

Received 17 April 2014; Accepted 17 April 2014; Published 28 April 2014

Copyright (c) 2014 Tongxing Li et al. This is an open access article distributed under the Creative Commons Attribution License, which permits unrestricted use, distribution, and reproduction in any medium, provided the original work is properly cited.

Differential, difference, and dynamic equations are used for modeling many problems arising in the engineering and natural sciences. Therefore, analysis of qualitative properties of solutions to such equations is crucial for applications. It is important to develop new theories and methods, as well as to modify and refine the well-known techniques, for the analysis of new classes of problems.

We received 11 papers for possible publication. Five papers published in this special issue address a number of challenging problems. The special issue opens with the contribution by Professor R. P. Silva who gives the characterization of the limiting behavior of solutions of elliptic equations driven by the $p$-Laplacian operator with Neumann boundary conditions posed in a family of thin domains.

Professors Z. Luo et al. consider a class of generalized $n$ species Lotka-Volterra type and Gilpin-Ayala type competition systems with multiple delays and impulses. Using Krasnoselskii fixed point theorem in a cone of Banach space, they derive some verifiable necessary and sufficient conditions for the existence of positive periodic solutions of the previously mentioned. As applications, some special cases of the previous system are examined and some earlier results are extended and improved.

Professors M. Ünal and Y. N. Raffoul use the fixed point theory to investigate the qualitative analysis of a nonlinear delay dynamic equation on an arbitrary time scale. They illustrate new results by applying them to various kinds of time scales.

In the paper by Professor A. Reinfelds, the conjugacy of a discrete semidynamical system and its partially decoupled discrete semidynamical system in a Banach space is proved in a neighbourhood of the nontrivial invariant manifold.

The contribution by Professors S. H. Da Silva et al. closes this special issue. They show normal hyperbolicity property of curves of equilibria and prove the continuity of global attractors for the flow of a class of nonlocal evolution equations with respect to some functional parameter.

We hope that this collection of papers will attract interest of researchers working in related areas and will stimulate further progress in the Qualitative Theory of Differential, Difference, and Dynamic Equations.

\section{Acknowledgments}

We would like to express our sincere gratitude to the authors for their contributions. We are also thankful to the reviewers whose professional comments and valuable suggestions guaranteed the high quality of these selected papers. Finally, Tongxing Li would like to acknowledge the Guest Editors Tuncay Candan and Ethiraju Thandapani for their selfless assistance and guidance in producing this special edition within the specified timeframe.

Tongxing $\mathrm{Li}$

Tuncay Candan Ethiraju Thandapani 


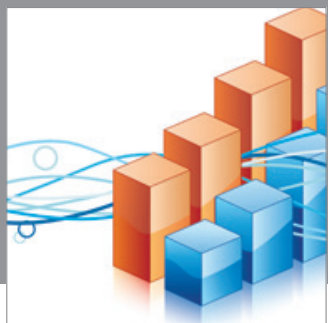

Advances in

Operations Research

mansans

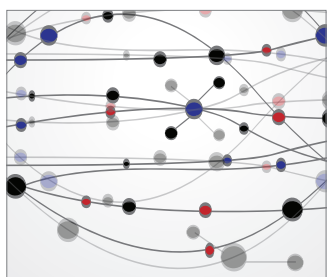

The Scientific World Journal
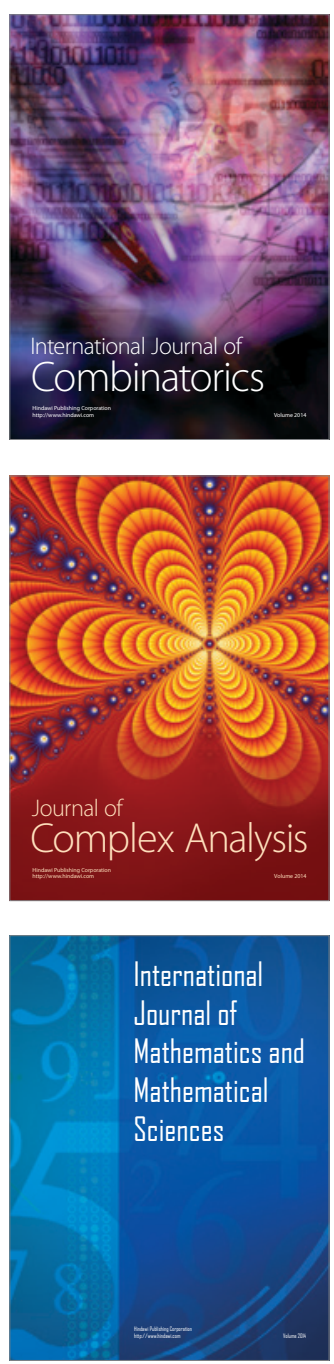
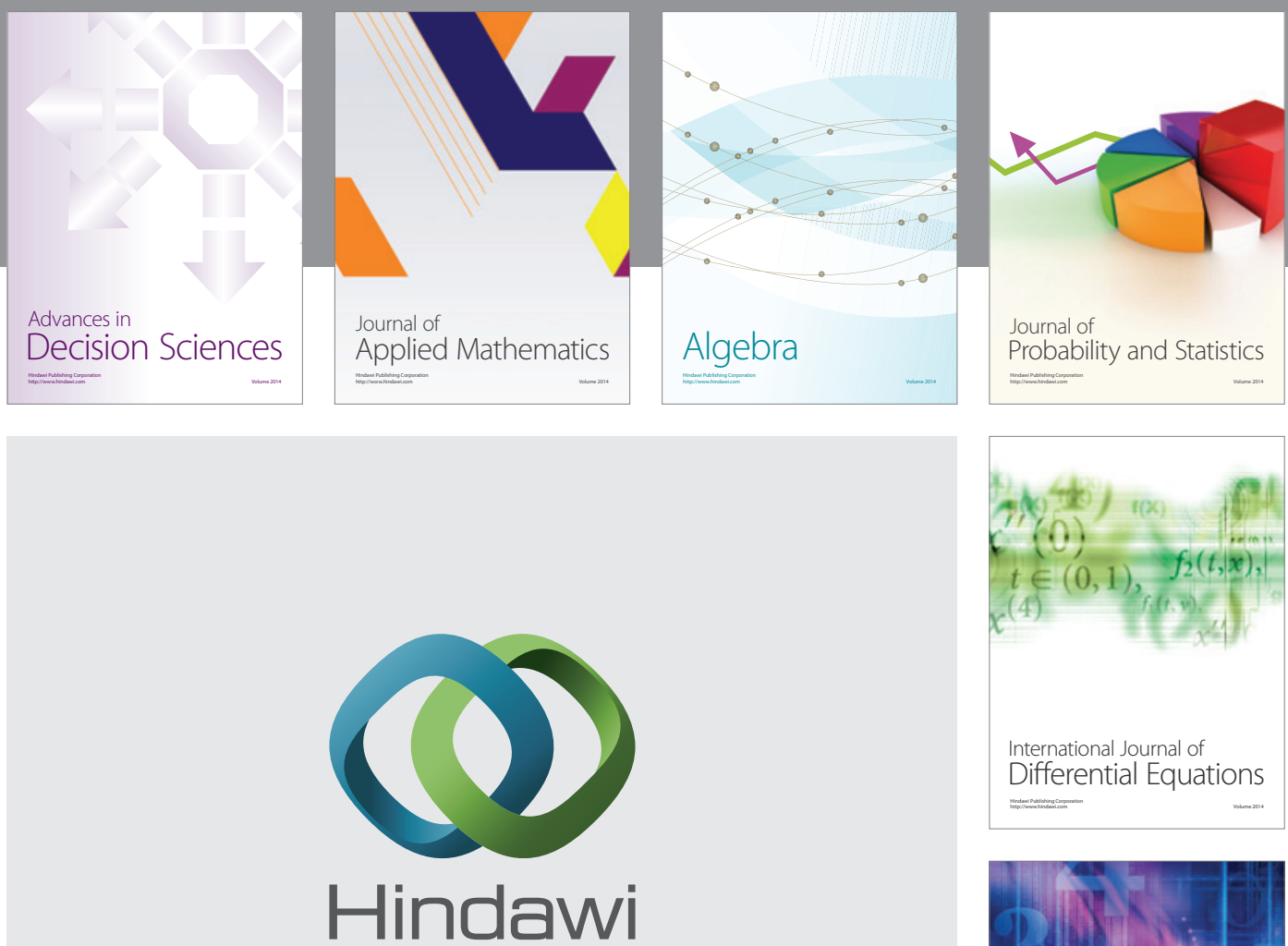

Submit your manuscripts at http://www.hindawi.com
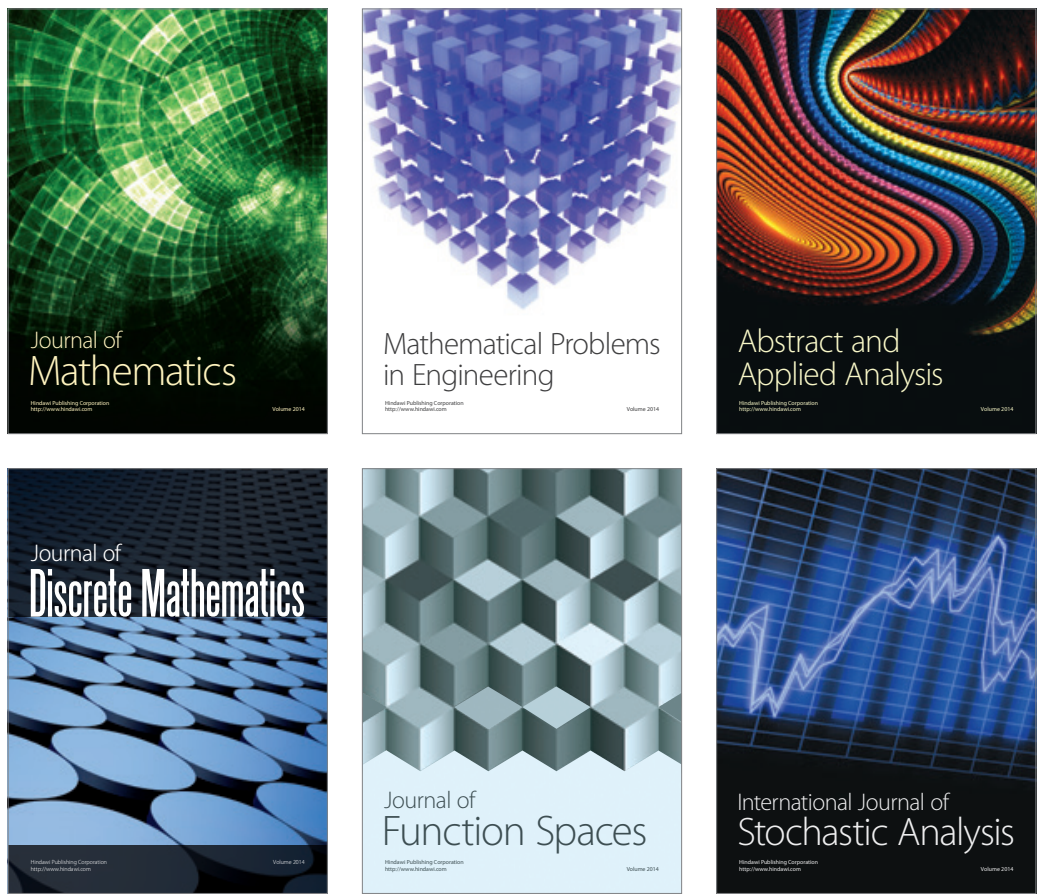

Journal of

Function Spaces

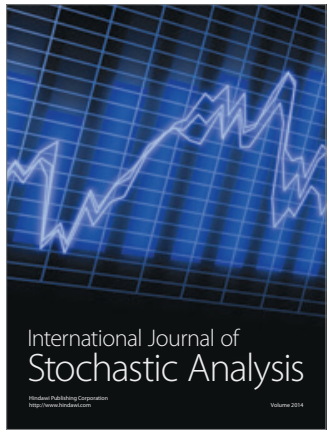

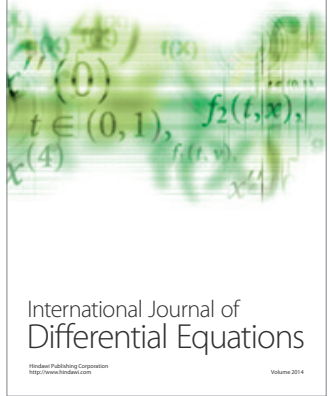
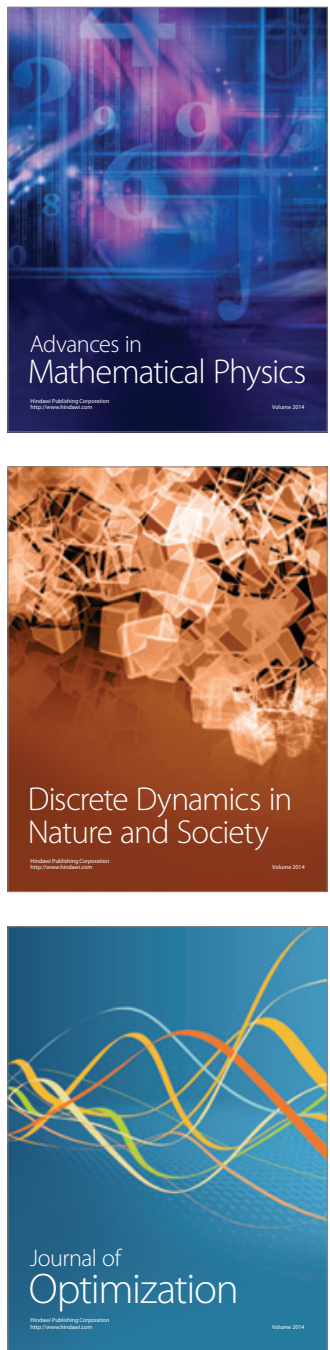